Mathematical Programming manuscript No.

(will be inserted by the editor)

Matteo Fischetti · Andrea Lodi

\title{
Optimizing over the first Chvátal closure
}

Received: July 28, 2005 / Revised version:

\begin{abstract}
How difficult is, in practice, to optimize exactly over the first Chvátal closure of a generic ILP? Which fraction of the integrality gap can be closed this way, e.g., for some hard problems in the MIPLIB library? Does it pay to insist on rank-1 Chvátal-Gomory inequalities until no such inequality is violated, or one should better follow the usual strategy of generating (mixed-integer) Gomory cuts of any rank from the optimal tableau rows? How effective is this general-purpose approach for solving matching problems, where the first Chvátal closure coincides with the integer hull? Can this approach be useful as a research (off-line) tool to guess the structure of some relevant classes of inequalities, when a specific combinatorial problem is addressed? In this paper we give, for the first time, concrete answers to the above questions, based on an extensive computational analysis. Our approach is to model the rank-1 ChvátalGomory separation problem, which is known to be NP-hard, through a MIP model, which is then solved through a general-purpose MIP solver. As far as we know, this approach was never implemented and evaluated computationally by previous authors, though it gives a very useful separation tool for general ILP problems. We report the optimal value over the first Chvátal closure for a set of ILP problems from MIPLIB 3.0. We also report, for the first time, the optimal solution of a very hard instance from MIPIB 2003, namely nsrand-ipx, obtained by using our cut separation procedure to preprocess the original ILP model. Finally, we describe a new class of ATSP facets found with the help of our separation procedure.
\end{abstract}

Key words: integer programs, separation problems, Chvátal-Gomory cuts, computational analysis.

\section{Introduction}

We consider the Integer Linear Program (ILP) problem

$$
\min \left\{c^{T} x: A x \leq b, x \geq 0 \text { integer }\right\}
$$

where $A$ is a $m \times n$ matrix, $b \in \mathbb{R}^{m}$, and $c \in \mathbb{R}^{n}$, along with the two associated polyhedra:

$$
\begin{aligned}
& P:=\left\{x \in \mathbb{R}_{+}^{n}: A x \leq b\right\} \\
& P_{I}:=\operatorname{conv}\left\{x \in Z_{+}^{n}: A x \leq b\right\}=\operatorname{conv}\left(P \cap Z^{n}\right)
\end{aligned}
$$

DEI, University of Padova, via Gradenigo 6A - 35131 Padova - Italy e-mail: matteo.fischetti@unipd.it

DEIS, University of Bologna, viale Risorgimento 2 - 40136 Bologna - Italy IBM T.J. Watson Research Center, P.O. Box 218, Yorktown Heights, NY 10598 e-mail: alodi@us.ibm.com

Mathematics Subject Classification (1991): 90C06, 90C10, 90C11, 90C27, 90C59 
Unless explicitly stated, we assume that $(A, b)$ is an integer matrix. Moreover, we assume that $P_{I}$ is strictly contained in $P$, i.e., that $P$ has fractional vertices.

A Chvátal-Gomory $(C G)$ cut $[12,7]$ is a valid inequality for $P_{I}$ of the form

$$
\left\lfloor u^{T} A\right\rfloor x \leq\left\lfloor u^{T} b\right\rfloor,
$$

where $u \in R_{+}^{m}$ is called the $C G$ multiplier vector, and $\lfloor\cdot\rfloor$ denotes lower integer part. Note that $\mathrm{CG}$ cuts depend on $P$ and not directly on $P_{I}$, i.e., different formulations $A x \leq b, x \geq 0$ of the same integer problem can produce different CG cuts. Inequality (4) is said of rank 1 with respect to formulation $A x \leq b$, $x \geq 0$; CG cuts of rank $h \geq 2$ are obtained in a similar way, starting with an enlarged system containing all CG cuts of rank less than $h$. It is known [16] that undominated CG cuts only arise for rational CG multipliers $u_{i} \in[0,1)^{m}$, provided that $(A, b)$ is integer.

The first Chvátal closure of $P$ is defined as

$$
P_{1}:=\left\{x \geq 0: A x \leq b,\left\lfloor u^{T} A\right\rfloor x \leq\left\lfloor u^{T} b\right\rfloor \text { for all } u \in \mathbb{R}_{+}^{m}\right\}
$$

A basic result, due to Chvátal [7], is that $P_{1}$ is indeed a polyhedron, i.e., a finite number of CG cuts suffice to define it.

Clearly, $P_{I} \subseteq P_{1} \subseteq P$. It was shown by Gomory [12,13] that every fractional vertex $x^{*}$ of $P$ associated with a certain basis $B$ (say) of $(A, I)$ can be cut off by the CG cut in which $u$ is chosen as the $i$-th row of $B^{-1}$, where $i$ is the row associated with any fractional component of $x^{*}$. Therefore, $P_{1} \subset P$ in case $P \neq P_{I}$, i.e., $P_{1}$ gives a better approximation of $P_{I}$ than $P$. In some cases, one has that $P_{I}=P_{1}$ as, e.g., for matching problems where undominated CG cuts correspond to the famous Edmonds' blossom inequalities [8,16]. Even in case $P_{I} \subset P_{1}$, however, we are interested in optimizing $c^{T} x$ over $P_{1}$ in order to get a hopefully tight lower bound on the optimal value of the original ILP problem (1).

By the well-known equivalence between optimization and separation [16], we will address the $C G$ separation problem in which we are given any point $x^{*} \in \mathbb{R}^{n}$ and we ask for a hyperplane separating $x^{*}$ from $P_{1}$ (if any). Without loss of generality we can assume that $x^{*} \in P$, since all other points can be cut by simply enumerating the members of the original inequality system $A x \leq b$, $x \geq 0$. Therefore, the separation problem we are actually interested in, called $C G$-SEP in the sequel, reads:

Definition 1. (CG-SEP) Given any point $x^{*} \in P$ find (if any) a $C G$ cut that is violated by $x^{*}$, i.e., find $u \in \mathbb{R}_{+}^{m}$ such that $\left\lfloor u^{T} A\right\rfloor x^{*}>\left\lfloor u^{T} b\right\rfloor$, or prove that no such $u$ exists.

It was proved by Eisenbrand [10] that CG-SEP is NP-hard, so optimizing over $P_{1}$ also is. It is worth observing that this result is not in contrast with the fact that any vertex of $P$ can easily be cut by a CG cuts read from the associated tableau. Indeed, the equivalence between optimization and separation heavily depends on the use of the ellipsoid method for linear programming, whose convergence in 
a polynomial number of iterations requires to consider a sequence of points $x^{*}$ generated as centers of appropriate ellipsoids, so the point $x^{*}$ to be cut off cannot be assumed to have any special property such as, e.g., being integer, or being a vertex of $P$-just for the same reason for which the convergence of binary search in a logarithmic number of steps requires to consider the middle point of the current interval at each step (considering any other point can affect the logarithmic convergence of the method). Therefore the "trick" of replacing $x^{*}$ by a nearby vertex of $P$ does not work, even if this operation can be performed in polynomial time, since the polynomial convergence of the overall ellipsoid method would be affected. On the other hand, the use of a cutting plane method based on the simplex method will guarantee that the point $x^{*}$ to be cut at any iteration is a vertex (of the current formulation), but this approach can generate an exponential number of Gomory cuts (some of which even of rank larger than 1 ), so this method cannot lead to an efficient approach for optimizing over $P_{1}$ (besides the fact that the simplex algorithm itself is non-polynomial).

In this paper we address the issue of evaluating the practical strength of $P_{1}$ in approximating $P_{I}$. Our approach is to model the rank-1 CG separation problem as a MIP model, which is then solved through a general-purpose MIP solver. As far as we know, this approach was never implemented (and evaluated computationally) by previous authors ${ }^{1}$. In Section 2 we describe our MIP model for CG separation. In Section 3 we investigate computationally the practical (and theoretical) benefits derived from using our separation tool. In particular, we report the optimal value over the first Chvátal closure for a set of problems from MIPLIB 3.0 [4]. We also report, for the first time, the optimal solution of a very hard instance from MIPIB 2003 [1], namely nsrand-ipx, obtained by using our CG separation procedure to preprocess the original ILP model. Finally, we describe a new class of ATSP facets found with the help of our CG separation procedure.

An extended abstract of the present paper appeared in the proceedings of the IPCO XI meeting [11].

\section{How?}

The first question we address is how to solve the NP-hard CG-SEP problem.

\subsection{A MIP model}

Given the input point $x^{*} \geq 0$ to be separated, CG-SEP calls for a CG cut $\alpha^{T} x \leq$ $\alpha_{0}$ which is (maximally) violated by $x^{*}$, where $\alpha=\left\lfloor u^{T} A\right\rfloor$ and $\alpha_{0}=\left\lfloor u^{T} b\right\rfloor$ for a certain $u \in \mathbb{R}_{+}$. A first CG-SEP model then reads:

1 The fact that CG separation can be modeled as a MIP can instead be attributed to folklore and has been recently pointed out, e.g., by Caprara and Letchford [5] for the related class of split cuts. 


$$
\begin{aligned}
\max & \alpha^{T} x^{*}-\alpha_{0} \\
& \alpha_{j} \leq u^{T} A_{j}, \quad \text { for } j=1, \cdots, n \\
& \alpha_{0}>u^{T} b-1 \\
& u_{i} \geq 0, \quad \text { for } i=1, \cdots, m \\
& \alpha, \alpha_{0} \quad \text { integer }
\end{aligned}
$$

In the above model, the integer variables $\alpha_{j}$ and $\alpha_{0}$ play the role of coefficients $\left\lfloor u^{T} A_{j}\right\rfloor$ and $\left\lfloor u^{T} b\right\rfloor$ in the CG cut, respectively. Hence the objective function (6) gives the amount of violation of the CG cut evaluated for $x=x^{*}$, that we want to maximize. Because of the sign of the objective function coefficients, the rounding conditions $\alpha_{j}=\left\lfloor u^{T} A_{j}\right\rfloor$ can be imposed though upper bound conditions on variables $\alpha_{j}(j=1, \cdots, n)$, as in $(7)$, and with a lower bound condition on $\alpha_{0}$, as in (8). Note that this latter condition is stated as a strict inequality so as to avoid an integer $u^{T} b$ be rounded to $u^{T} b-1$.

We will now elaborate the basic model above, in the attempt of reducing its size while improving the quality of the generated CG cuts. We observe that any variable $x_{j}$ with $x_{j}^{*}=0$ gives no contribution to the cut violation, hence there is no need to consider it explicitly in the separation model. Indeed, whenever $x_{j}^{*}=0$ one can simply disregard $x_{j}$ in the separation problem, and recompute the corresponding coefficient a posteriori, when the optimal $u$ has been determined, as $\alpha_{j}:=u^{T} A_{j}$ (no time-consuming lifting operations being needed). The same holds for variables at their upper bound in $x^{*}$, which can be complemented before separation (an operation that affects the right-hand-side vector $b$ ).

In addition, as already mentioned, one can assume $u_{i}<1$ in case the $i$-th row of $(A, b)$ is integer. Indeed, the CG cut associated with any $u_{i} \geq 1$ is easily seen to be a dominated one, as it can be obtained as the sum of the CG cut with $u_{i}$ replaced by its fractional part, plus $\left\lfloor u_{i}\right\rfloor$ times the $i$-th constraint $a_{i}^{T} x \leq b_{i}$. (Some unexpected practical implications of imposing the above multiplier upper bound will be discussed in Section 2.2.)

In view of the considerations above, we propose the following MIP model for CG-SEP:

$$
\begin{aligned}
\max & \sum_{j \in J\left(x^{*}\right)} \alpha_{j} x_{j}^{*}-\alpha_{0} \\
& f_{j}=u^{T} A_{j}-\alpha_{j}, \quad \text { for } j \in J\left(x^{*}\right) \\
& f_{0}=u^{T} b-\alpha_{0} \\
& 0 \leq f_{j} \leq 1-\delta, \quad \text { for } j \in J\left(x^{*}\right) \cup\{0\} \\
& 0 \leq u_{i} \leq 1-\delta, \quad \text { for } i=1, \cdots, m \\
& \alpha_{j} \text { integer, } \quad \text { for } j \in J\left(x^{*}\right) \cup\{0\}
\end{aligned}
$$

where $J\left(x^{*}\right):=\left\{j \in\{1, \cdots, n\}: x_{j}^{*}>0\right\}$ denotes the support of $x^{*}$ (possibly after having complemented same variables and updated $b$ accordingly). In this 
model we introduced explicit slack variables $f_{j}=u^{T} A_{j}-\left\lfloor u^{T} A_{j}\right\rfloor$ to capture the coefficient fractionality, and required them to be in range $[0,1-\delta]$ for a small $\delta>0$. (Some of these constraints are in fact redundant because of the objective function, but we observed that their incorporation into the model improves its numerical behavior.) In our implementation we chose the $\delta=0.01$; this choice improves the numerical stability of our method, though it could affect the exact nature of our CG separation procedure in some pathological cases.

Model (11)-(16) can easily be adapted to deal with the case where the original model (1) contains free variables and/or equations-in this latter case, condition (15) is replaced by

$$
-1+\delta \leq u_{i} \leq 1-\delta .
$$

In addition, one can also allow for the presence of continuous variables ${ }^{2}$. Indeed, if a variable $x_{j}$ is not restricted to be integer, one can still derive a valid cut by setting $\alpha_{j}=0$ and imposing $u^{T} A_{j} \geq 0$ (or $u^{T} A_{j}=0$ in case $x_{j}$ is a free variable).

\subsection{Multiplier Selection}

Model (11)-(16) typically has several equivalent solutions, hence we are interested in a clever way of breaking ties. Indeed, our ultimate goal is to find, for each fractional point $x^{*}$ to be cut off, a "round" of Chvátal-Gomory cuts that are significantly violated and whose overall effect is as strong as possible in improving the current LP relaxation. Moreover, the separation problem should be solved as quickly as possible. In this view, we have implemented the following additional mechanisms to guide the MIP solver to provide effective cuts.

1. Making the cut sparser (and stronger). A major practical issue with CG separation is the strength of the returned cuts. As a matter of fact, several equivalent solutions of the separation problem typically exist, some of which produce very weak cuts for the ILP model (1). This is because the separation problem actually considers the face $F\left(x^{*}\right)$ of $P_{I}$ where all the ILP constraints that are tight at $x^{*}$ (including the variable bounds) are imposed as equalities. Hence for this face there exist several formulations of a same cut, which are equivalent for $F\left(x^{*}\right)$ but not for $P_{I}$. One therefore has to consider the issue of producing a violated cut that is as strong as possible with respect to $P_{I}$. In practice, this leads to the issue of producing "minimal" CG multiplier vectors with as few nonzero entries as possible. To achieve minimality, we modified the objective function (11) into

$$
\max \left(\sum_{j \in J\left(x^{*}\right)} \alpha_{j} x_{j}^{*}-\alpha_{0}\right)-\sum_{i=1}^{m} w_{i} u_{i}
$$

2 The mixed-integer case is however not covered in the original Chvátal's work, and would be more appropriately addressed in the context of Gomory mixed-integer cuts. 
by introducing the penalty term $-\sum_{i} w_{i} u_{i}$, where $w_{i}=10^{-4}$ for all $i$. It is worth observing that, because of equations (12)-(13), one can rewrite the violation term $\sum_{j \in J\left(x^{*}\right)} \alpha_{j} x_{j}^{*}-\alpha_{0}$ in (11) as $f_{0}-\sum_{j \in J\left(x^{*}\right)} f_{j} x_{j}^{*}-\sum_{i=1}^{m} u_{i} s_{i}^{*}$, where $s^{*}=b-A x^{*} \geq 0$. As a consequence, the penalty term $w_{i}$ is only relevant for tight constraints, and can be omitted when $s_{i}^{*}>0$.

2. Enhancing the "collaborative behavior" of the cuts. Preliminary computational experiments have shown the presence of the lower/upper bounds (15) or (17) on the multipliers $u_{i}$ sometimes weaken the overall performance of our method. This is true, in particular, for the constraints stated in equality form-removing the conditions $-1<u_{i}<1$ generally produces much better results, though these bounds were only meant at choosing a (sparse but) equivalent formulation of a same violated cut. A possible explanation of this quite surprising behavior is that removing the lower/upper bounds on the CG multiplier is beneficial for the MIP heuristics we use (ILOG-Cplex 9.1). Even more importantly, it seems that leaving more freedom in the choice of the multipliers produces a beneficial "diversification effect" in breaking ties between equivalent solutions of the separation model (11)-(16), hence each call of the MIP separation tends to return cuts that are less correlated with those generated in the previous calls. This results into a better "collaborative behavior" of the generated cuts (also well known, though not completely understood, when using rounds of Gomory cuts read from the tableau). Therefore, in our implementation we allow for the maximum degree of freedom, and remove all the lower/upper bound conditions (15) or (17) on the CG multipliers (we only keep the nonnegativity conditions associated with constraints in less-than-or-equal-to form, as they are required for the correctness of the method). The underlying idea is that, for slack cuts with $s_{i}^{*}>0$, the objective function already tends to reduce the absolute value of the associated multiplier, hence the lower/upper bounds on the multiplier are somehow redundant. For tight constraints, instead, imposing the bound conditions is immaterial as far as the separation of the current point $x^{*}$ is concerned, but hurts the MIP heuristics as it reduces their degree of freedom. A drawback of this choice is however that (in spite of the penalty terms in the objective function) the returned cuts may involve multipliers $u_{i}$ with $\left|u_{i}\right|>1$, i.e., the cuts may be unnecessarily dense (and quite weak, in case of less-than-or-equal-to constraints). We therefore implemented a simple cut post-processing procedure where each CG multiplier returned by the MIP solver is replaced by its fractional part (when this is mathematically correct) before deriving the actual CG cut. As shown in the computational tests, this mechanism turns out to be quite effective, the main so for the problems involving a large number of equality (i.e., always tight) constraints.

\subsection{Implementation in a pure cutting-plane framework}

We have embedded our CG cut separator into a pure cutting-plane framework where we keep generating violated CG cuts of rank 1 (with respect to the original 
formulation of the ILP model at hand), until we stop either because no such violated cut exists (in which case we have optimized over the first closure), or because a time-limit condition is met. In our prototype implementation, we avoided a number of possible improvements consisting, e.g., in designing ad-hoc heuristics for our MIP separation, or in driving the MIP solver to produce a sequence of CG cuts with disjoint support. We only used the following simple mechanisms:

- When the LP relaxation of the original ILP model (1) is solved, we take all the violated Gomory fractional cuts that can be read from the current tableau, and skip CG separation; in principle this mechanism could also be applied in the subsequent iterations, provided that we skip the Gomory cuts having a non-zero $\mathrm{CG}$ multiplier for a constraint that was not in the original formulation, so as to ensure that all generated cuts are of rank 1.

- The MIP solver for CG separation is invoked with an initial lower bound of 0.01, meaning that we are only interested in CG cuts violated by more than 0.01 .

- Each time the MIP solver for CG separation updates its incumbent solution, we store the corresponding CG cut in a pool, with the aim of adding it to the current ILP formulation right after the end of the separation phase. Because of the penalty term that appears in the MIP objective function (11), however, we found that it is useful to skip all the CG cuts with the same violation $\sum_{j \in J\left(x^{*}\right)} \alpha_{j} x_{j}^{*}-\alpha_{0}$, except the one with the sparsest support.

- The MIP execution for CG separation is stopped if either the optimal solution has been found, or a prefixed number $\tau$ of branching nodes has been explored after the last update of the incumbent solution, where $\tau=1000$ if the violation of the incumbent is less than 0.2 , and $\tau=100$ if the violation is greater or equal to 0.2 .

\section{Why?}

We next try to give concrete (i.e., supported by computational results) answers to some important questions related to the practical behavior of our MIP separator and to the theoretical effectiveness of rank-1 CG cuts, when applied to a wide range of ILP instances - both with and without a specific combinatorial structure.

Our implementation of the cutting-plane method uses the commercial software ILOG-Cplex 9.1 as the LP solver, whereas the separation problem is solved through ILOG-Cplex 9.1 MIP solver with "mip emphasis 4" parameter; see [14]. All computing times refer to a Pentium M 1.7 Ghz notebook with 512 MByte RAM. 


\subsection{Can we solve matching problems?}

In order to develop and test our code in a "familiar" setting, we addressed first the Edmonds-Johnson [9] matching problem, defined as:

$$
\min \left\{c^{T} x: A x=b, 0 \leq x \leq q, x \text { integer }\right\}
$$

where $A, b, q$ are integer arrays of appropriate dimensions, and $\sum_{i=1}^{m}\left|a_{i j}\right| \leq$ 2 holds for all $j=1, \cdots, n$. A celebrated result of Edmonds [8,9] says that $P_{1}$ is an integer polyhedron, i.e., $P_{1}$ and $P_{I}$ coincide for matching problems. Moreover, undominated Chvátal cuts (called blossom inequalities) only arise for $u \in\{0,1 / 2\}^{m}$, and correspond to setting $u_{i}=1 / 2$ for (a) the equations $a_{i}^{T} x=b_{i}$ associated with a certain set $H$ (say) and (b) the upper-bound constraints $x_{j} \leq$ $q_{j}$ associated with a possibly-empty set $T \subseteq\{1, \cdots, n\}$, such that $\sum_{i \in H} b_{i}+$ $\sum_{j \in T} q_{j}$ is an odd number. Blossom inequalities can be separated in polynomial time by an ad-hoc procedure looking for a minimum-weight odd cut is a certain graph; see Padberg and Rao [17] and Letchford, Reinelt and Theis [15].

A relevant case of matching problems arises when $A$ is the node-edge incidence matrix of a (multi-)graph $G=(V, E)$. Given edge costs $c_{e}(e \in E)$ and node values $b_{v} \geq 0(v \in V)$, we call for a minimum-cost partial graph $G^{\prime}=(V, M)$ of $G$ where each node $v$ has degree $b_{v}$. The $b$-matching problem can then be formulated as

$$
\min \left\{c^{T} x: \sum_{e \in \delta(v)} x_{e}=b_{v} \text { for } v \in V, 0 \leq x \leq 1, x \text { integer }\right\}
$$

Also belonging to the family of matching problems is the $T$-join problem, where each node $v$ has an associated parity $b_{v} \in\{0,1\}$ and one looks for a minimumcost partial graph $G^{\prime}=(V, M)$ of $G$ where each node $v$ has an odd degree if and only if $b_{v}=1$. This problem can be formulated as the $b$-matching problem above, with the degree equations replaced by $\sum_{e \in \delta(v)} x_{e}-2 z_{v}=b_{v}, z_{v}$ integer, for all $v \in V$.

Table 1 reports computational results on a set of 2-matching problems taken from TSPLIB: in all cases, our method was able to compute the optimal solution over the first Chvátal closure, and produced an integer solution - as expected.

Table 2 shows that the multiplier-selection rules discussed in Section 2.2 are indeed effective in reducing the overall computing time. In the table, we compare the results of our best algorithm (v.best) with two alternative versions in which the mechanism for making the cuts sparser (v.dense) or more collaborative (v.bound) has been disabled. For each run, a limit of 100 separation calls has been imposed. The table clearly shows the performance deterioration - in particular, when imposing the multiplier bounds.

It should be observed that some of these instances can be solved in a much shorter computing time by just applying ILOG-Cplex $9.1 \mathrm{MIP}$ solver to the rank0 model (20). However, for some hard instances our method also has a practical interest if used in a cut-and-branch approach where we abort our cut generation after the addition a certain number of CG cuts, and then switch to a commercial 


\begin{tabular}{l|rrrrr} 
ID & initial LB & Optimum & \# iter.s & \# cuts & CPU time \\
\hline eil101 & 619.0 & 623.0 & 17 & 34 & 0.95 \\
gr120 & $6,662.5$ & $6,694.0$ & 38 & 49 & 6.14 \\
pr124 & $50,164.0$ & $51,477.0$ & 83 & 141 & 13.96 \\
gr137 & $66,643.5$ & $67,009.0$ & 15 & 37 & 1.26 \\
pr144 & $32,776.0$ & $33,652.0$ & 35 & 71 & 3.86 \\
ch150 & $6,281.0$ & $6,337.0$ & 76 & 125 & 44.41 \\
rat195 & $2,272.5$ & $2,297.0$ & 125 & 231 & 160.74 \\
kroA200 & $27,053.0$ & $27,426.0$ & 26 & 81 & 5.56 \\
kroB200 & $27,347.0$ & $27,768.0$ & 171 & 332 & 215.37 \\
ts225 & $115,605.0$ & $121,261.0$ & 613 & 1401 & $2,914.36$ \\
pr226 & $55,247.5$ & $57,177.0$ & 230 & 319 & 166.83 \\
gr229 & $127,411.0$ & $128,353.0$ & 96 & 176 & 87.37 \\
gil262 & $2,222.5$ & $2,248.0$ & 150 & 304 & 195.89 \\
a280 & $2,534.0$ & $2,550.0$ & 89 & 129 & 34.89 \\
lin318 & $38,963.5$ & $39,266.0$ & 321 & 589 & $4,046.93$ \\
\hline
\end{tabular}

Table 1. 2-Matching Problems.

\begin{tabular}{l|rrrr|rrrr|rrrr} 
& \multicolumn{4}{|c|}{ v.best } & \multicolumn{4}{|c}{ v.dense: denser cuts } & \multicolumn{4}{c}{ v.bound: $u_{i} \in[-0.99,0.99]$} \\
\cline { 2 - 12 } & \# & \# & CPU & \% gap & \# & $\#$ & CPU & \% gap & \# & $\#$ & CPU & \% gap \\
ID & iter.s & cuts & time & closed & iter.s & cuts & time & closed & iter.s & cuts & time & closed \\
\hline eil101 & 17 & 34 & 1.03 & 100.0 & 17 & 34 & 1.06 & 100.0 & 100 & 217 & 40.60 & 92.6 \\
gr120 & 38 & 49 & 6.45 & 100.0 & 42 & 62 & 12.88 & 100.0 & 100 & 277 & 104.50 & 83.4 \\
pr124 & 83 & 141 & 14.57 & 100.0 & 93 & 134 & 22.52 & 100.0 & 100 & 210 & 41.40 & 25.6 \\
gr137 & 15 & 37 & 1.37 & 100.0 & 15 & 37 & 1.42 & 100.0 & 100 & 221 & 62.00 & 77.2 \\
pr144 & 35 & 71 & 4.29 & 100.0 & 38 & 79 & 8.05 & 100.0 & 100 & 231 & 46.50 & 65.5 \\
ch150 & 76 & 125 & 45.91 & 100.0 & 81 & 154 & 75.26 & 100.0 & 100 & 276 & 111.90 & 57.7 \\
rat195 & 100 & 171 & 79.20 & 97.4 & 100 & 194 & 104.40 & 93.3 & 100 & 292 & 167.90 & 34.8 \\
kroA200 & 26 & 81 & 6.21 & 100.0 & 26 & 81 & 6.34 & 100.0 & 100 & 250 & 87.50 & 80.3 \\
kroB200 & 100 & 175 & 39.60 & 82.2 & 100 & 171 & 39.20 & 77.0 & 100 & 229 & 87.70 & 30.9 \\
ts225 & 100 & 172 & 51.70 & 84.1 & 100 & 179 & 54.00 & 88.8 & 100 & 245 & 102.00 & 37.6 \\
pr226 & 100 & 170 & 33.90 & 96.1 & 100 & 152 & 31.40 & 80.8 & 100 & 195 & 73.50 & 31.3 \\
gr229 & 96 & 176 & 92.60 & 100.0 & 100 & 220 & 145.70 & 97.4 & 100 & 249 & 156.40 & 46.3 \\
gi1262 & 100 & 194 & 59.20 & 97.0 & 100 & 202 & 103.10 & 96.7 & 100 & 247 & 112.30 & 48.5 \\
a280 & 89 & 129 & 39.47 & 100.0 & 70 & 100 & 33.84 & 100.0 & 100 & 176 & 102.00 & 50.0 \\
lin318 & 100 & 169 & 73.30 & 68.0 & 100 & 183 & 93.90 & 69.4 & 100 & 273 & 210.20 & 26.6 \\
\hline
\end{tabular}

Table 2. 2-Matching Problems: impact of the multiplier selection rules.

MIP solver for concluding the optimization. This is illustrated in Table 3, where some large 2-matching instances are solved through ILOG-Cplex MIP solver with and without the addition of an initial pool of CG cuts generated by 100 calls to our separation procedure.

\subsection{How tight is the first closure for MIPLIB instances?}

Table 4 reports the outcome of our experiments on a test-bed made by all pure-integer problems from MIPLIB 3.0 [4]. The cases where our cutting plane procedure exceeded a time limit of 3 hours are given in the bottom part of the table. In this situation, the percentage of integrality gap closed (\% gap closed $)$, computed as 100-100(opt_value $\left(P_{I}\right)$-opt_value $\left.\left(P_{1}\right)\right) /\left(\right.$ opt_value $\left(P_{I}\right)-$ opt_value $(P))$, is underestimated as we only have a lower bound on the optimal value opt_value $\left(P_{1}\right)$ over the first closure. 


\begin{tabular}{|c|c|c|c|c|c|c|c|c|}
\hline \multirow[b]{2}{*}{ ID } & \multicolumn{3}{|c|}{ ILOG-Cplex } & \multicolumn{5}{|c|}{ cut-and-branch } \\
\hline & $\begin{array}{l}\text { \% gap } \\
\text { closed }\end{array}$ & nodes & time & \# cuts & $\begin{array}{l}\text { \% gap } \\
\text { closed }\end{array}$ & $\begin{array}{r}\text { separation } \\
\text { time }\end{array}$ & nodes & $\begin{array}{l}\text { total } \\
\text { time }\end{array}$ \\
\hline kroB200 & 100.0 & 330,913 & $2,748.24$ & 179 & 77.1 & 39.20 & 348 & 60.67 \\
\hline ts225 & 47.1 & 230,115 & $1 \mathrm{~h}$ & 193 & 85.9 & 46.10 & 1,018 & 75.39 \\
\hline $\operatorname{pr} 226$ & 55.0 & 288,901 & $1 \mathrm{~h}$ & 168 & 91.3 & 31.60 & 51 & 35.20 \\
\hline gr229 & 100.0 & 15,005 & 180.79 & 182 & 96.9 & 54.70 & 7 & 60.94 \\
\hline gil262 & 100.0 & 117,506 & $2,094.77$ & 172 & 97.4 & 54.50 & 1 & 58.39 \\
\hline $\operatorname{lin} 318$ & 53.3 & 117,100 & $1 \mathrm{~h}$ & 182 & 69.5 & 62.60 & 24,734 & 814.74 \\
\hline
\end{tabular}

Table 3. 2-Matching Problems: cut-and-branch approach.

\begin{tabular}{|c|c|c|c|c|c|}
\hline ID & Optimum & \# iter.s & \# cuts & $\begin{array}{l}\% \text { gap } \\
\text { closed }\end{array}$ & time \\
\hline air03 & $340,160.00$ & 1 & 35 & 100.0 & 0.65 \\
\hline gt2 & $21,166.00$ & 192 & 529 & 100.0 & 239.93 \\
\hline lseu & $1,120.00$ & 98 & 195 & 91.3 & $1,516.90$ \\
\hline mitre & $115,155.00$ & 935 & 1,128 & 100.0 & $1,995.15$ \\
\hline $\bmod 008$ & 307.00 & 29 & 118 & 100.0 & 12.25 \\
\hline $\bmod 010$ & $6,548.00$ & 5 & 40 & 100.0 & 0.74 \\
\hline nw04 & $16,862.00$ & 127 & 492 & 100.0 & 509.27 \\
\hline p0033 & $3,089.00$ & 42 & 115 & 85.4 & 16.20 \\
\hline p0548 & $8,691.00$ & 587 & 1,809 & 100.0 & $1,679.76$ \\
\hline stein 27 & 18.00 & 142 & 392 & 0.0 & 521.00 \\
\hline air04 & $56,137.00$ & 129 & 830 & $\geq 28.9$ & $3 \mathrm{~h}$ \\
\hline air05 & $26,374.00$ & 212 & 1,196 & $\overline{\geq} 30.4$ & $3 \mathrm{~h}$ \\
\hline cap6000 & $-2,451,377.00$ & 176 & 295 & $>23.4$ & $3 \mathrm{~h}$ \\
\hline enigma & 0.00 & 13,234 & 53,635 & -- & $3 \mathrm{~h}$ \\
\hline fast0507 & 174.00 & 21 & 299 & $\geq 4.7$ & $3 \mathrm{~h}$ \\
\hline fiber & $405,935.18$ & 432 & 1,880 & $\geq 98.8$ & $3 \mathrm{~h}$ \\
\hline harp2 & $-73,899,798.00$ & 1,077 & 1,549 & $\geq 43.9$ & $3 \mathrm{~h}$ \\
\hline 1152lav & $4,722.00$ & 793 & 2,249 & $\geq 60.0$ & $3 \mathrm{~h}$ \\
\hline $\operatorname{misc} 03$ & $3,360.00$ & 428 & 1,154 & $\geq 51.0$ & $3 \mathrm{~h}$ \\
\hline $\operatorname{misc} 07$ & $2,810.00$ & 618 & 1,870 & $\geq 16.9$ & $3 \mathrm{~h}$ \\
\hline p0201 & $7,615.00$ & 911 & 2,568 & $\overline{\geq} 60.6$ & $3 \mathrm{~h}$ \\
\hline p0282 & $258,411.00$ & 641 & 1,920 & $\geq 99.9$ & $3 \mathrm{~h}$ \\
\hline p2756 & $3,124.00$ & 1,018 & 4,358 & $\overline{\geq} 90.4$ & $3 \mathrm{~h}$ \\
\hline seymour & 423.00 & 94 & 3,383 & $\geq 23.5$ & $3 \mathrm{~h}$ \\
\hline stein 45 & 30.00 & 14,463 & 31,643 & $\overline{\geq} 0.0$ & $3 \mathrm{~h}$ \\
\hline
\end{tabular}

Table 4. General IPs of the MIPLIB 3.0. Note that for instance gt2 in 192 iterations the bound is improved to reach the optimal value but no integer solution can be found in the time limit of 3 hours.

The impact of the mechanisms for making the cuts sparser and more collaborative, as described in Section 2.2, is in the MIPLIB context less impressive. In particular, the diversification effect guaranteed by removing the multiplier lower/upper bounds appears crucial just for a few specific instances. For example, if one does not remove the multiplier bounds when solving problem mitre, the first 100 separations are completely ineffective in improving the lower bound value of $114,821.48$ obtained after the addition of the Gomory cuts read from the initial tableau-though 355 new cuts are added. As a comparison, the version without multiplier bounds brings the lower bound to $114,865.26$ after the same number of iterations, i.e., it closes $13.2 \%$ of the gap. In general, however, the overall impact is not as strong as the one shown in Table 2 for the 2-matching 
case. One possible explanation is that the models in the MIPLIB are more heterogeneous (in terms of constraints) and involve fewer equations with respect to the 2-matching ones, hence our diversification mechanism becomes less important. The use of the penalty terms for making the cuts sparser appears instead quite crucial for the MIPLIB instances too. For example, for problem fiber our best version closes $91.0 \%$ of the initial gap after 100 iterations, while the version without the penalty terms closes only $33.9 \%$ of the gap in the same number of iterations. This trend is consistently confirmed on the other instances.

Finally, in order to evaluate the viability of using our cut generator in a cut-and-branch approach akin to the one we described for 2-matching problems, we made the following experiment on the very hard ILP instance harp2. After having generated 211 rank-1 CG cuts (53 of which are tight at the end of the cutting-plane phase) in 100 rounds of separation, we switched to ILOG-Cplex MIP solver and obtained the optimal solution within 1,500 CPU seconds and $400 \mathrm{~K}$ nodes (including both cut generation and branching), while ILOG-Cplex alone required more than 15,000 CPU seconds and 7M nodes to solve the original instance.

\subsection{Beyond the first closure?}

The previous experiments show that optimizing over the first closure often gives a very tight approximation of the integer optimal value, though it may require a large computing time. Besides the obvious issue of designing more specific separation procedures for CG cuts, we addressed the possibility of using our cutting plane method as a pre-processing tool, to be used to strengthen the user's formulation by possibly exploiting cuts of Chvátal rank larger than 1. This idea was evaluated by comparing two different cut preprocessors, namely:

- cpx: Apply ILOG-Cplex 9.1 (with mip emphasis "move best bound") on the current ILP model, save the final root-node model (including the generated cuts) in a file, and repeat on the new model until a total time limit is exceeded.

- cpx-cg: Apply ILOG-Cplex 9.1 (with mip emphasis "move best bound") on the current ILP model, followed by 600 seconds of our CG separation procedure; then save in file the ILP model with all the cuts that are active in the last LP solution ${ }^{3}$, and repeat on the new model until a total time limit is exceeded.

Figure 1 compares the behavior of the two methods above on a very hard instance from MIPLIB 2003, namely timtab1 (viewed here as a pure ILP problem, as it is correct in this case). The horizontal axis counts the number of calls to the separation procedures (not the computing time nor the number of generated cuts), hence comparing the graph slopes can be misleading since every separation call produced about 10-50 cuts for $c p x$, but just 1-5 cuts for $c p x-c g$. Method $c p x$

3 As we no longer insist in staying within the first Chvátal closure, CG cuts could be improved by deriving the stronger Gomory mixed-integer cut from the surrogate equations $u^{T} A+s=u^{T} b, s \geq 0$. 
exhibits an early tailing-off phenomenon, while the growth for $c p x$ - $c g$ is more regular and produces a much better final lower bound.

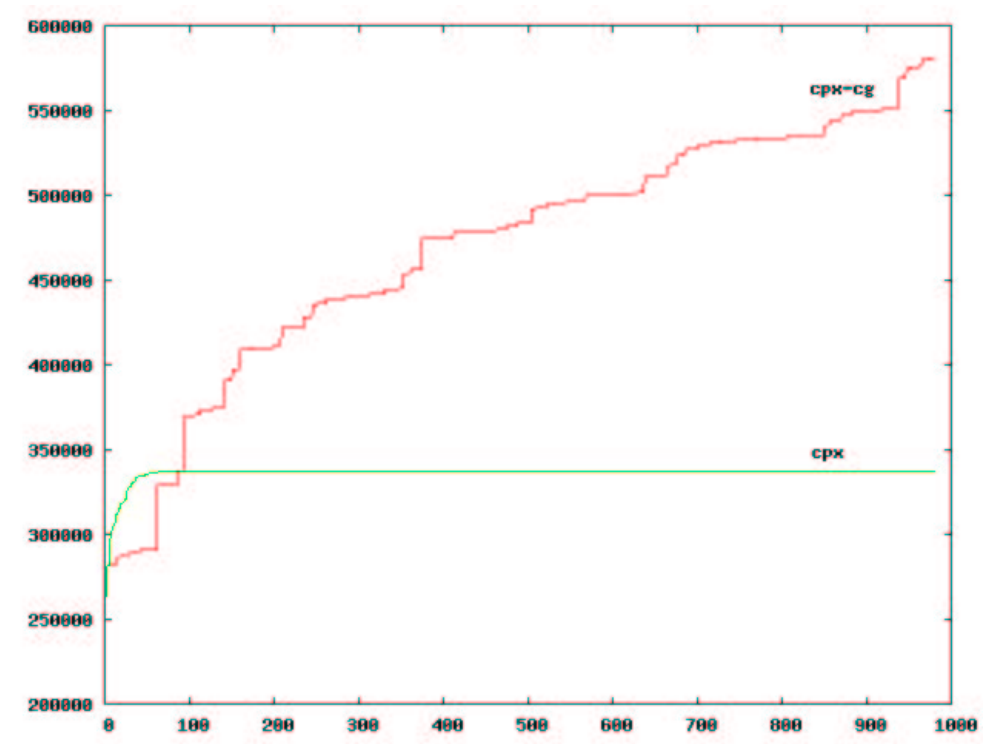

Fig. 1. Lower bounds provided by $c p x$ and $c p x-c g$ after each call of the separation procedures, for the hard MIPLIB instance timtab1.

Remarkably, our cut preprocessor allowed us to find, for the first time [1], a provable optimal solution of value $51,200.00$ for the very hard instance nsrandipx (in order to get a pure ILP, the objective function $x 1$ was replaced by its expression given by the first constraint in the model.) To this end, we applied ILOG-Cplex 9.1 MIP solver to the tightened formulation returned by $c p x-c g$ after 4800 seconds-this brought the initial LP bound from 49,667.89 to 50,665.71.

\subsection{Can we discover new classes of strong inequalities?}

The capability of separating over the CG cut class also has an interesting application in the off-line study of the facial structure of a specific problem. This is in the spirit of the widely-used approach of producing (through appropriate software such as PORTA [6]) an explicit polyhedral description of very small instances of the problem at hand, but has the advantage of being applicable to instances of much larger size.

To illustrate a possible application to the Asymmetric Travelling Salesman Problem (ATSP), we made the following experiment. We set up a partial ATSP formulation including out- and in-degree equations, plus the subtour elimination constraints on 2-node sets, i.e., we addressed the NP-hard Asymmetric Assignment Problem (AAP) relaxation studied by Balas [2]. We then applied our separation procedure to a specific ATSP instance from TSPLIB, namely the 48-node 
instance $r y 48 p$, and stored the CG cuts we found along with the associated CG multipliers ${ }^{4}$. One of the returned $\mathrm{CG}$ cut had a violation of 0.75 , and was associated with the following non-zero CG multipliers (reported in parenthesis): out-degree equation for nodes $21(-0.5), 35(0.5), 47(-0.25), 2(0.5), 26(0.5)$, $12(-0.25), 20(-0.25)$, and $48(-0.5)$; in-degree equations for nodes $5(-0.5), 29$ $(-0.5), 35(-0.5), 47(0.25), 4(-0.5), 42(-0.5), 32(0.5), 33(0.25)$, and $20(0.25)$; subtour elimination constraint on the 2-node sets $\{5,29\}(0.5),\{21,47\}(0.25)$, and $\{35,45\}(0.5)$. To simplify our analysis, we normalized the corresponding cut by adding 1 to all the negative CG multipliers, thus producing an equivalent CG cut in the so-called $h$-canonical form [3], i.e., with non-negative and relative prime coefficients, whose support leaves at least one node $h$ isolated. This form immediately showed the presence of clones [3], that for the purpose of our analysis could be removed from the digraph. After renaming the nodes, we were left with a 8-node digraph and with the following CG multipliers: out-degree equation for nodes $1(0.75), 3(0.75), 4(0.5), 5(0.75)$, and $6(0.5)$; in-degree equations for nodes $1(0.25), 2(0.5), 5(0.25), 6(0.5)$, and $7(0.25)$; subtour elimination constraint on the 2-node sets $\{4,5\}(0.25)$ and $\{6,7\}(0.5)$. The associated CG cut is $\alpha^{T} x \leq \alpha_{0}$, where

$$
\alpha=\left(\begin{array}{llllllll}
0 & 1 & 0 & 0 & 1 & 1 & 1 & 0 \\
0 & 0 & 0 & 0 & 0 & 0 & 0 & 0 \\
1 & 1 & 0 & 0 & 1 & 1 & 1 & 0 \\
0 & 1 & 0 & 0 & 1 & 1 & 0 & 0 \\
1 & 1 & 0 & 1 & 0 & 1 & 1 & 0 \\
0 & 1 & 0 & 0 & 0 & 0 & 1 & 0 \\
0 & 0 & 0 & 0 & 0 & 1 & 0 & 0 \\
0 & 0 & 0 & 0 & 0 & 0 & 0 & 0
\end{array}\right) ; \alpha_{0}=5
$$

and it is easily shown (by computational methods) to be facet-defining for ATSP (and hence for AAP). Using clique lifting [3] we can then obtain a large class of ATSP facets, that to the best of our knowledge is new.

\section{Acknowledgements}

Work partially supported by MIUR and CNR, Italy, and by the EU project ADONET. Thanks are due to Jon Lee and François Margot for useful discussions.

\section{References}

1. T. Achterberg, T. Koch, A. Martin, The mixed integer programming library: MIPLIB 2003, http://miplib.zib.de.

2. E. Balas, The Asymmetric Assignment Problem and some new facets of the Traveling Salesman Polytope on a directed graph, SIAM Journal on Discrete Mathematics 2, 425451, 1989 .

4 For CG cuts in the form returned by our procedure, these multipliers could easily be recomputed a posteriori, by solving an LP. 
3. E. Balas, M. Fischetti, A lifting procedure for the Asymmetric Traveling Salesman Polytope and a large new class of facet, Mathematical Programming 58, 325-352, 1993.

4. R.E. Bixby, S. Ceria, C.M. McZeal, M.W.P. Savelsbergh, MIPLIB 3.0, http://www. caam.rice.edu/ bixby/miplib/miplib.html.

5. A. Caprara, A.N. Letchford, On the separation of split cuts and related inequalities, Mathematical Programming 94, 279-294, 2003.

6. T. Christof, A. Löbel, PORTA - POlyhedron Representation Transformation Algorithm, http://www.zib.de/Optimization/Software/Porta/.

7. V. Chvátal, Edmonds polytopes and a hierarchy of combinatorial problems, Discrete Mathematics 4, 305-337, 1973.

8. J. Edmonds, Maximum matching and a polyhedron with $\{0,1\}$-vertices, J. Res. Nat. Bur. Standards B 69, 125-130, 1965.

9. J. Edmonds, H.L. Johnson, Matching: a well-solved class of integer linear programs, in R.K. Guy et al. (ed.s), Combinatorial Structures and Their Applications, Gordon and Breach, New York, 89-92, 1970.

10. F. Eisenbrand, On the membership problem for the elementary closure of a polyhedron, Combinatorica 19, 297-300, 1999.

11. M. Fischetti, A. Lodi, Optimizing over the first Chvátal closure, in M. Jünger and V. Kaibel (ed.s), Integer Programming and Combinatorial Optimization - IPCO 2005, LNCS 3509, Springer-Verlag, Berlin Heidelberg, 12-22, 2005.

12. R.E. Gomory, Outline of an algorithm for integer solutions to linear programs, Bulletin of the $A M S 64,275-278,1958$.

13. R.E. Gomory, An algorithm for integer solutions to linear programs, in R.L. Graves and P. Wolfe (ed.s) Recent Advances in Mathematical Programming, McGraw-Hill, New York, 1963.

14. ILOG Cplex 9.1: User's Manual and Reference Manual, ILOG, S.A., http://www.ilog.com/, 2005.

15. A.N. Letchford, G. Reinelt, D.O. Theis, A faster exact separation algorithm for blossom inequalities, in D. Bienstock and G. Nemhauser (ed.s), Integer Programming and Combinatorial Optimization - IPCO 2004, LNCS 3064, Springer-Verlag, Berlin Heidelberg, 196-205, 2004.

16. G.L. Nemhauser, L.A. Wolsey, Integer and Combinatorial Optimization, Wiley, New York, 1988.

17. M.W. Padberg, M.R. Rao, Odd Minimum Cut-Sets and b-Matchings, Mathematics of Operations Research 7, 67-80, 1982. 